\title{
Attitudes and knowledge of clinicians and dieticians towards cancer nutrition in a tertiary referral oncology centre
}

\author{
M. Khan ${ }^{1}$, A. Vickers ${ }^{1}$, Z. Kordatou ${ }^{1}$, M. Abraham ${ }^{2}$ and W. Mansoor ${ }^{1}$ \\ ${ }^{1}$ Department of Oncology, The Christie NHS Foundation Trust, Manchester, M20 4BX and \\ ${ }^{2}$ Department of Dietetics, The Christie NHS Foundation Trust, Manchester, M20 4BX, UK
}

Despite advances in therapeutic options for cancers, treatment is often impeded by the presence of cancer attributable malnutrition and metabolic derrangements ${ }^{(1)}$. To increase early recognition and intervention of malnutrition and metabolic derangements in cancer, organisations such as the European Society for Clinical Nutrition and Metabolism (ESPEN) have emphasised a greater need for cancer nutritional risk screening and education on cancer nutrition ${ }^{(2)}$. However, prior studies have shown a lack of understanding of fundamental aspects of nutrition screening and treatment by physicians ${ }^{(3)}$ but such data is lacking with regards to physician's knowledge in cancer malnutrition and metabolic derangements. Therefore, we investigated and compared the attitudes and knowledge towards nutritional support in cancer between physicians and dieticians at a tertiary referral oncology centre.

Physicians (categorised as foundation year 2 (FY2s), core trainees (CTs), specialist registrars (SpRs), clinical research fellows (CRFs) and consultant oncologists (COs)) and oncology dieticians (ODs) were invited to participate in a multiple-choice questionnaire test. Questions assessed the attitudes towards cancer nutrition, assessment and management of common cancer nutritional scenarios. Comparisons in terms of correct and incorrect responses were made between physicians to ODs. Data is presented as the mean \pm standard error (SE) and percentage test score with the use of one-way analysis of variance (ANOVA) to determine if any observed differences were statistically significant ( $\mathrm{p}$-value $<0.05)$.

\begin{tabular}{|c|c|c|c|c|c|c|c|c|}
\hline \multirow{2}{*}{ Test Score } & & FY2s $(n=15)$ & CTs $(n=8)$ & SpRs $(n=13)$ & CRFs $(n=13)$ & $\operatorname{COs}(n=30)$ & ODs $(n=10)$ & All physicians $(\mathrm{n}=79)$ \\
\hline & Mean & 6.6 & 10.1 & 7.62 & 6.38 & 8.33 & 18.6 & 7.73 \\
\hline & SE & 1.34 & 1.77 & 1.17 & 1.32 & 0.929 & 1.06 & 0.553 \\
\hline & $\%$ & 27.5 & 42.1 & 31.8 & 26.6 & 34.6 & 77.5 & 32.2 \\
\hline
\end{tabular}

Maximum test score $=24$. Total sample size $=89$. All physicians vs. ODs, $p<0.0001 ;$ FY2s vs. ODs, $p<0.0001 ;$ CTs vs. ODs, $p=0.0036$; CRFs vs. ODs, $p<0.0001$; SpRs vs ODs, $\mathrm{p}<0.0001$; COs vs. ODs, $\mathrm{p}<0.0001$. No statistical significant differences in test scores between individual physician groups.

Physicians collectively and as individual groups achieved a statistically significant lower score compared to ODs, thereby demonstrating a poorer knowledge base in topics centred upon cancer malnutrition and metabolic derangements. No statistically significant differences were observed between test scores when comparing physician groups despite an anecdotal perceived increase in clinical exposure and acumen with increasing seniority. Additionally, only $41.8 \%(n=33)$ of physicians collectively felt they had adequate knowledge of nutritional support for cancer patients despite $69.6 \%(\mathrm{n}=55)$ stating they regularly make decisions regarding nutritional support in cancer patients. Overall, $89.9 \%(\mathrm{n}=71)$ stated they felt provision of support in cancer nutrition would be valuable to their clinical career demonstrating a need for further cancer malnutrition and metabolic derangements education amongst physicians.

1. Hebuterne X, Lemarie E, Michallet M, de Montreuil CB, Schneider SM, Goldwasser F (2014) Prevalence of malnutrition and current use of nutrition support in patients with cancer. JPEN J Parenter Enteral Nutr 38, 196-204.

2. Arends J, Bachmann P, Baracos V et al. (2017) ESPEN guidelines on nutrition in cancer patients. Clin Nutr 36, 11-48

3. Mowe M, Bosaeus I, Rasmussen HH et al. (2008) Insufficient nutritional knowledge among health care workers? Clin Nutr 27, $196-202$. 